\title{
A scientific perspective of neijin (internal strength)
}

\begin{abstract}
The investigation of Neijin (Internal Strength) via its manifestation in body motion forges a theoretical link between Qi and muscle actions in the regulation of motion to comport with the principles of yin and yang. This gives a formulation of neijin in the framework of physics and physiology and provides a pragmatic qi-motion paradigm to train for the strength, as well as a window into Qi at a level of basic science. This paper grew from a presentation given on the occasion of the author receiving the Scientist of the Year Award at the $17^{\text {th }}$ World Congress on Qigong, Tai Chi, and TCM, Sep 3, 2016, San Francisco.
\end{abstract}

Volume 5 Issue 3 - 2017

\section{C.P Ong}

Tai Chi Instructor/Author, USA

Correspondence: C.P. Ong, Tai Chi Instructor/Author, USA, Email cpiiong@gmail.com

Received: November 26, 2016 | Published: February 6, 2017

\section{Introduction}

The practice of Taijiquan develops a kind of core strength, which by traditional theory is differentiated from regular physical strength, called Internal Strength or Neijin. Neijin is not a well formulated concept, which is why references to in-depth studies of the subject are hard to find even in the Chinese literature on Taijiquan. There is no clear consensus of what it is or how best to train for it among experts of the various schools Taijiquan. But it is exalted as the mysterious strength that underlies the prowess of Chinese Martial Arts - the stuff that seems to defy one's sense of speed, strength and power expected in combat.

The rule of thumb of the uncharacteristic strength is that the more it appears of "physical force" the less it is of neijin. There is a persistent viewpoint that neijin is not associated with the musculature, so much so that it has spawned a cultural disdain of muscles in Taijiquan. This paper seeks to reconcile the musculature with neijin by looking at neijin feats as described in the classics of Taijiquan, such as the skill of "four ounces repelling a thousand pounds." However, the study does not include the controversial claim of "empty space force," which purportedly can propel a person by the wave of a hand without physical contact, and notes only that the phenomenon has not withstood scrutiny.

Neijin is presented in the Taiji garb of yin and yang, and its development is shrouded in the concepts of spirit (Shen), mind (Yi), and life-force energy (Qi), which are foreign to the musculoskeletal structure. These are based on the Taiji theory of yin and yang, which though ancient, still pervades everything Chinese, and they form the basic framework of Traditional Chinese Medicine (TCM). Qi is fundamental and central to both Taijiquan and TCM.

The advantage in formulating neijin over TCM methods, such as acupuncture, is that there is a tangible and concrete representation of neijin that can be studied. Incredible as the hidden strength of neijin may be, its kungfu prowess arises from body motion, and therefore is subject to biomechanics. The investigation forges a theoretical connection between the role of Qi in neijin through body motion, and physics and physiology at a basic level. In this regard, the paper is following the lead of Shin Lin (University of California, Irvine), who has published several papers, pioneering the study of Qi at the level of basic science.

Specifically, this paper looks at Qi and the yin-yang principles that underpin neijin as manifested in the musculoskeletal framework, in the harmony of the motion of the many segments of the body. This harmony is reduced to the concept of inner balance - the balance and alignment of the internal movements at the joints and the axial core. The concept of inner balance establishes a paradigm of Qi and muscle actions, where Qi grows in association with the cultivation of an ideal kind of motion, namely, that which comports with the yinyang principles.

The upshot is that the principle of inner balance endows the body with an ease of motion change internally at the joints and an unforced and smooth flow of motion through the body without resistance. Imbued with inner balance, the body responds spontaneously with the captivating wonders of neijin. The body maintains balance and structural integrity when interacting with an external agent, such as in combat. And the many segments of the body move in tandem, unifying the body's momentum to produce a maximal impact of force.

The import of the qi-motion paradigm is that it establishes a discipline of muscle actions to conform to the principle of inner balance in the quest of the Holy Grail of neijin. It does so by cultivating Qi associated with the factors of inner balance, and then uses the Qi medium to generate the ideal body motion. Neijin arises from this ideal body motion. In other words, the formulation provides a scientific basis for neijin. The additional prize is that it provides another window to the study of Qi at the level of basic science, an importance that cannot be underestimated as Qi forms the central tenet of TCM.

\section{What is neijin?}

Neijin $\square \square$ is the wondrous stuff behind the prowess of the kungfu of Taijiquan. This strength is differentiated from regular physical strength, which is $l i \square$ in Chinese. The Chinese character for this strength is jin $\square$, which left radical conveys that it is a highly cultured and trained li $\square$; the prefix nei $\square$ of neijin indicates its internal character.

What fascinates is that the kungfu application appears effortless with hardly any physical exertion of force-hidden so to speak. It seems that Physics is turning on its head-the "weaker" overcoming the "stronger" and the "slower" beating the "faster."

What fuels its mystery is that the ethos of Taijiquan resides in the internal, not characterized by external attributes of strength, like that of muscle bulk or the vigor of physical activities that we are familiar with. Indeed, the only thing extraordinary about Taijiquan masters is that they are distinguished by the ordinariness of their physique. The gentle and slow motion that defines the art cannot be more remote from the speed and power of a knockout punch. That is the mystique of Taijiquan's neijin. So what Taiji practitioner or martial artist would not harbor the dream of developing the magic of neijin? 
For a quick answer of neijin, we can paraphrase Chen Xiaowang's explanation in an equation:

$$
\text { Neijin }=\text { Muscle actions }+ \text { Qi }
$$

In explaining neijin, Grandmaster Chen Xiaowang says that the strength of Qi alone is not great, and the strength of muscle force without Qi is crude and inflexible.

The answer is encouraging as it indicates that one is building a store of neijin in the very practice of Taijiquan: The practice nurtures Qi and doing the movements entails muscle actions. For that matter, all oriental martial arts train to develop Qi (Ki in Japanese) as well.

But where is the stuff of neijin? Many have practiced Taijiquan for years without getting it. Not every Taijiquan practitioner can tap into neijin to beat back a bully. Neijin is illusive. That is what makes the quest of this soft strength of neijin fascinating and challenging.

So the quick answer of neijin needs a lot of supplementals in exposition. How does Qi meld in with muscle actions? A good place to start is at the waist. We use the waist to generate power actions in sports and martial arts, as well as at work, hence the term waist power. But waist power does seem mundane in light of the grandiose of neijin intimated. That may be so, but think about it - we ruefully fall far short of our potential to produce the waist power that the body is capable of, and we seem limited in our ability to improve upon it in training. Weekend golfers know well the limits of the range of their drives.

In fact, Taijiquan recognizes that the main source of neijin power lies in the waist region. The Chinese term for it is more specific, called dang-yao jin $\square \square \square$,which translates as waist-groin power. The terminology indicates that the power is derived from the pairing of the actions of the groin (dang) and waist (yao).

The eminent status of the waist-groin region is enshrined in the canons of Taijiquan. ${ }^{1}$ The classical literatures are replete with references to the waist-groin as the center of control and the main source of power of neijin.

Control center is at the waist...

Zhu zai yu yao xing yu shou zhi $\square \square \square \square \square \square \square \square$

Mind intent commands, motion originates at the waist region

Ming yi yuan tou zai yao ji $\square \square \square \square \square \square \square$

Be attentive at all times to the waist region

Ke ke liu xin zai yao jian $\square \square \square \square \square \square \square$

Relax completely the abdomen and qi soars

Settle the tail bone centrally, and the spirit rises to the top

Fu nei song jing qi teng ran $\square \square \square \square \square \square \square$

\section{Wei lv zhong zheng shen guan ding $\quad \square \square \square \square \square \square$}

Mind as the commander, qi as the signal flag, and waist as the banner

\section{Xin wei ling qi wei qi yao wei dao $\square \square \square \square \square \square \square \square \square$}

The study of waist power through the lens of Taijiquan shines the light of science at neijin as it manifests in the body, and opens up the esoteric Taiji yin-yang theory and the role of qi in the musculoskeletal framework. Generating waist power requires the play of the pelvic platform, called the kua $\square$. Thus, Taijiquan places the greatest emphasis on the kua in practice.

\section{The biomechanics of waist power}

In generating waist power, the upper body rotates in one direction, and the lower body turns in the opposite direction in mutual support. Where should the division of the rotational motions between the upper and lower body be? If only the shoulder and chest were turning to power the upper body action, the rest of the muscles below would be underutilized, a common flaw.

If the division of the rotations occurred at the knees, then the muscle mass below would have to support a much larger mass above, which would cause injuries to the knees or ankles. The division at the kua junction represents the most proportionate distribution of muscle masses between the upper and lower body, which can be seen in the power actions of Figure 1.

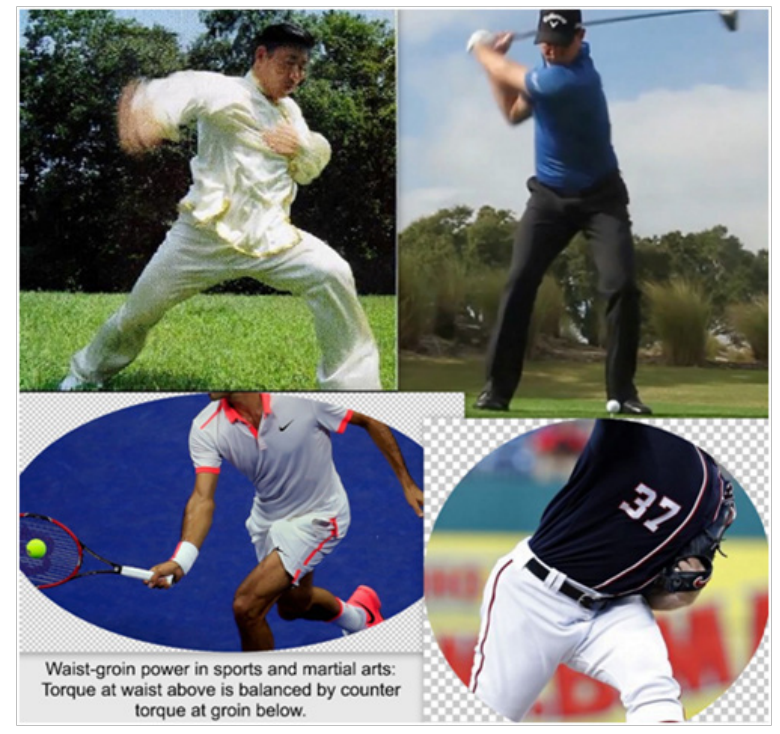

Figure I Rotational motions at the waist-groin junction.

There is another crucial anatomical factor to waist power. The vertebral column ends at the sacrum which sits on the pelvic (iliac) base at a "plane" joint, called the sacral-iliac joint (SIJ). The SIJ forms the hub of the transfer of forces between the upper and lower body via the three levers, the spine and the legs through the pelvic platform. ${ }^{2}$

The hub function of the SIJ has actually been described in the Taiji classics as well:

If the body cannot maneuver to take timely advantage ( $d e$ ji de shi $\square \square \square \square$ ), then the problem is at the waist and legs.

Strength is released from the spine, supported by the legs, stepping with body following in accord.

Li you ji fa bu sui shen huan $\square \square \square \square \square \square \square \square$

\section{How does the body comprehend force?}

Force arises when there is a change in motion. The change in body motion that we have cognition of occurs in two ways. The first is the contractile actions of muscles, which produce body motion. We are not cognitive of the contractile force of the muscles but we have cognition of the motion. The second is the change in body motion wrought by an external agent, for example, when we run into a wall or 
are hit by a moving object. We have direct cognition of the force that results from this change, often from the injuries sustained.

We may associate force with the speed of the fist but we are not conscious of the biceps and triceps contracting and working in antagonistic pairs to produce the motion. But what is the force of the punch? We appreciate the force imparted when it inflicts a bloody nose - in the impact of the collision between the head and fist. But there would be much less drama if the fist struck a pillow instead. So the force of a punch is not the same; it depends on what is struck.

The force that the body comprehends is the force that results from a change in its motion, more precisely, its momentum (mass x velocity). This is expressed by Newton's Second Law of Motion:

Average Force $=$ Change in Momentum/Time duration of the change.

From the Law, the key to producing more force is to generate more momentum by increasing speed or involving more body mass. However, it is complicated in practice as body motion is not uniform. Body motion is a composition of the movements of many segments. The issues of generating greater momentum involve several factors:

i. The body segments moving coherently in unison

ii. The alignment of muscle actions.

iii. The spinal rotation due to torsion by the Spinal Engine ${ }^{3}$

iv. The role of the fascia in force transmission.

In other words, the force that we produce - that which arises from a change in momentum - would be maximal if the internal momentum of the body is harmonized in unison. That is, generating strength is very much about regulating to harmonize body motion, not just about increasing body or muscle mass.

Here we study the movements of body segments and the alignment of muscle actions in generating momentum, but mention only in passing the other two factors, which will be topics of another paper. ${ }^{4}$

\section{Inner balance}

Taijiquan's game plan to generate greater momentum is to regulate the body segments to move in unison. The traditional theory couches this in the principle of harmony and articulates that the art be in accord with the Taiji principles of yin and yang, the main one of which is yinyang balance. This yin-yang harmony pervades everything Chinesein food, arts, music, fengshui, etc. In Traditional Chinese Medicine (TCM) health is a good store of the life-force energy, Qi, circulating in harmony.

The problem is that there is no quantitative analysis in Taiji theory to tell us what the balance of yin and yang is. However, in the manifestation of yin and yang, we can develop cognition of excesses or deficiencies of yin or yang, and hence of yin-yang imbalance. So in essence, Taijiquan methodology is cultivating the cognition of yin or yang in terms of deficiencies or excesses of muscle actions underlying body posture and motion. In Taijiquan training we are learning to discern yin-yang imbalances in the musculoskeletal framework.

In other words, we find the meat of yin-yang imbalance in the muscle actions underlying body motion and posture. We thus study muscle actions and qi dynamics and seek harmony between them in the context of generating dang-yao jin (waist power) to find and comprehend neijin. This is the modus operandi of Taijiquan to incorporate qi in the application of the yin-yang metaphysics to the art of body motion.

In this paper, we refer to the yin-yang balance of Taijiquan as inner balance, which is a state where the muscle actions underlying body posture or motion are not excessive (yang) or deficient (yin), independent of the loads on the body. Once we have cognition of the errors of muscle actions - excessive or deficient-we can work to resolve them towards a better state of balance. But, even as this points to a pragmatic approach, we encounter the practical problem of resolving imbalance. We cannot allocate so much muscle actions here and so much there to resolve the imbalance as in a scale balance nor are we cognitive of the muscle actions directly.

However, we can cultivate cognition and sensations of the effects of the errors of muscle actions. An instance of this is illustrated in a medical checkup where the doctor puts a stethoscope on your chest and asks that you breathe in. In doing so, the chest is heaved up, and the body becomes top-heavy, which falls easily with a gentle nudge. While the body is in physical balance, its inner balance is compromised - the abdomen is hollowed, weakening the support column of the midsection internally, and rendering the structure less able to keep balance. The body can learn from the top-heaviness as an effect of yin-yang imbalance of muscle actions.

We commonly introduce internal imbalance in the body structure in routine actions, such as when we raise a hand high up excitedly to attract attention. This shows that there are many combinations of muscle actions underlying a body posture and motion. For mundane activities the differences of the muscle support do not matter much, but in sports they determine the performance outcome. What should be the preferred combinations of muscle actions for a given body posture or motion? Taijiquan's answer is inner balance, namely, those combinations of muscle actions with lesser errors of yin-yang imbalance.

So training in Taijiquan is all about regulating body motion to harmonize the muscle actions to cultivate inner balance. The immediate import is a consolidation of the body's physical balance with a huge payoff of health benefits, which is well-studied. By reducing the errors, the muscle actions become better aligned and balanced, and thus the body's internal momentum becomes more harmonized. As we shall see, in the course of training, qi is also developed.

Think of the prize of inner balance thus: The force that ensues in any action is maximal and body balance remains intact. However, the problem of resolving the muscle actions still remains and of which we have no cognition. How do we get the body to listen?

\section{Neural responses}

The responses of neurobiology work very well for our bipedal balance and functionality, but not so when we need to summon the muscles necessary to power performance actions in sports. Golfers have the comparable muscle masses to deliver long drives, but train as hard as they like, few amateur players can improve significantly their golf range. We cannot at will elicit neural responses to fire the right combination of muscles. We cannot recruit the muscles directly even if we know which ones to recruit.

Moreover, the body is stubborn in its neural responses to recruit muscles, out of habits and convenience, which can bring harm to the body. For example, in picking up a box, the hands reach out and the body leans forward. The back muscles fire by reflex to keep the body from falling over in the leaning posture. In lifting the box, the weight 
pulls the body further down, requiring more muscle actions to keep balance. As a result, much of the muscle power goes to the reflex response to keep balance, and less to do the task at hand. There is no feedback of the debilitating effects of the muscle actions with regard to bad postures that can cause chronic backaches. One could move closer to the box, squat down to lift the box with better leverage with the aid of the leg muscles. The body can learn and incorporate from the discomfort of the strain as a feedback of the ill effects of posture due to muscle actions.

Similarly, in throwing a punch, the muscles closer to the fist tend to dominate. This dominance causes the arm to lunge forward, cutting the muscle power of the rest of the body to the action. The body again can learn to sense and associate the inadequacy of the punch due to the poor alignment and timeliness of the muscle activations.

Although we are presumed to have control of the voluntary movements in the somatic nervous system, we have no direct control-we have no communication with the muscles. The control we have is only at the command level, at the top hierarchy of the motor system. For instance, we have control at the command level, to touch the nose or ear, but we have no cognition of the multitude of neural activities in feedback and signal between the command and the innervation of the complex of muscles that result in the action.

There is a huge gap of neural activities between the command and the innervation of muscles that produce the motion at the bottom hierarchy. Training is at the mercy of this gap of neurobiology that we have no cognition of ${ }^{5}$. What sensory feedback data can we rely on in this gap to guide a preferred combination of muscle actions of the command?

To overcome this problem, Taijiquan resorts to the yin-yang theory to cultivate qi to bridge this gap via the yi-qi-motion paradigm:

\section{Yi dao qi dao qi dao shen dong $\square \square \square \square \square \square \square \square$}

Command arrives and activates qi; qi arrives and activates motion. In the response to the yi (mind) command at the top hierarchy, Taiji uses qi, developed in the training, to signal and guide the activation of muscles underlying the action or movement commanded. In other words, qi energy conveys the command to the muscle innervations that result in the movement (yi qi yun zhen $\square \square \square \square$ ), and to receive feedback data of the action. This signaling and sensory role of qi is developed by relating the nurturing of qi with the underlying muscle actions as they are being resolved towards inner balance, which is discussed next.

\section{Fangsong and qi}

Although we take $Q i \square$, the life-force energy, as given in Traditional Chinese Medicine (TCM), we can think of it as a composite of bioenergy, namely, any energy involved in biological functions and processes. There are bio-indicators that inform us of biological needs and functions. For instance, hunger is a biomarker associated with low food fuel, short of breath with low oxygen, cold with negative temperature gradient, pain with bodily harm and damage, feeling good with seratonin, etc. (emotions are more complicated, being triggered by a torrent of neural signals and hormonal changes). Thus, qi bioenergy becomes interesting if it can be associated with biomarkers. Then we can discern changes in the qi energy levels by their biomarkers.

Thanks to the pioneering work of Shin Lin and his research team, we can identify some bioenergy as surrogates of qi. They have quantified increases in the following physiological and bioenergetic changes due to Taijiquan or qi-energetics exercises: a) blood flow (perfusion), b) state of relaxation as indicated by heart-rate variability and brain-wave analysis, and c) bioenergy emission in the form of heat, light (photon counts), electrical charge, and conductance at acupuncture points. ${ }^{6}$

The first qi sensation of Taijiquan or qi-energetics exercise most commonly felt is tingling and warmth in the hands, due to increased blood flow or perfusion. However, Taijiquan relies more on changes in the bio-energy associated with the balance and alignment of muscle actions in the cultivation of inner balance. The initial sense of muscle actions is derived from their internal imbalances. The imbalances give rise to the experience of discomfort of tenseness or unease of motion.

To illustrate this imbalance at a basic level, extend an arm out and hold it in balance. The arm is in physical balance but the muscle actions supporting it can vary, for instance, when held stretched out or let to droop. Holding the arm up for ten minutes, tenseness and aches would set in, which indicates excessiveness in some muscle actions. Upon sensing the stress of discomfort, a symptom of the internal imbalance, the body triggers a reflex response of relaxation, which brings some relief.

This response is called fangsong, which is "to relax and let go." The reflex response, operationally, is a reset of the muscle activations, which improves the state of support with less stress - a consequence of the law of minimizing energy use in biology. This resettling into another combination of muscle actions is accompanied by an ease of flow of motion, and is sensed as qi energy, a biomarker of reduced tenseness.

The fangsong-relaxation response represents the rudiments of a tool, which we also call fangsong $\square \square$, that resolves the errors of internal imbalance of the muscle actions. Taijiquan practice develops this fangsong tool to reduce the errors of muscles actions, which equate to the nurturing of qi energy - the more the errors are reduced by fangsong, the more the qi.

The qi sensation is due primarily to a coherence of bio-energy in a better state of balance of muscle actions, derived from the lessening of the stress of tenseness or stiffness, and the ease of motion flow and transmission at the joints, together with a better blood flow and perfusion. These are mostly the somatosensory data from receptors in the ligaments, tendons, muscles, as well as from pressure and temperature of movements (kinesthesia) and receptors of position in space and of balance (proprioception).

\section{Inner and axial movements}

Taijiquan practice, motivated by dang-yao jin, is primarily concerned with the problem of the inner imbalance of the axial motion of the spine and the appendicular motion of the limbs, namely, the errors of excessive or deficient actions of the core axial muscles and the muscles of appendicular skeleton. Locally, at the joints, the concern is with the inner balance between the actions of the inner muscles that secure and stabilize the structure and the outer or prime-moving muscles that move the segments, namely, the internal movements.

We respond quite well and readily in the movements of the arms and legs, but are not very aware of the axial movements of the spine. We are not very clear of the muscle actions at the joints - the internal movements. The prime-moving appendicular and outer muscles tend to dominate in actions, which causes that of the internal and axial movements to lag. That is the main source of errors of muscle 
activations, which is what confounds training in the art of body motion.

The slow-motion and deliberative fangsong methodology works to rein in the dominant muscle actions, and allows the inner muscles to fire more so that the internal and axial movements can better align and balance in the actions. This induces more optimal muscle activations at higher levels, which leads to more coherent bioenergy. The higher activation levels of the muscles are often experienced as a surge of heat in practice, registered as qi. The body learns to associate the elevated qi energy as a biomarker of better balance and alignment of muscle actions. This association in time grows the qi as a more defining marker to regulate motion of inner balance.

In the stepping movements of Taijiquan practice the body settles stably in the substantial foot as it steps forward, say. One can see that compared to walking, the muscles are working at a higher level of activation in the weighting of the body in the solid foot and the slow pushing of the body forward. The relative increase in the bio-energy of Taijiquan play is measured by Shin Lin and his research team-the activation level of the quadricep muscles is found to be raised 2 to 4times more than in walking. ${ }^{7}$

To recapitulate, fangsong works in a passive mode - it resettles the muscle actions so that the outer muscles that move body segments become better aligned and balanced with the inner muscles that secure and stabilize the joints and structure. In this way, the errors of muscle actions that are too yang (excessive) or too yin (deficient) are reduced, and in conjunction, qi is cultivated under the varying load conditions of the body. The key point here is that the qi energy thus developed becomes a biomarker associated with the factors of fangsong resolution of muscle actions to seek inner balance. This point cannot be overemphasized, that the qi grows in time to sufficient maturity and is used as a marker to regulate Taijiquan motion via the yi-qi-motion paradigm.

\section{Regulating breath and fang song}

Regulating breath is very much a part of qi energetics. The Chinese character for breath is $q i \square$,the same as that for what is designated as bioenergy qi. They do seem to be different things, but the nurturing of qi must attend to breath. Indeed, the early literature refers to qi energetics as daoyin tu'na $\square \square \square \square$, which means "calisthenics and breath."

We may take breathing for granted, but we certainly have to attend to it when we run out of breath. Panting for breath is stressful, which clearly is not conducive to fangsong and qi nurturing. We slow down to catch our breath, a natural way to regulate it. We check our breath so that we do not overexert. We regulate our breath rhythmically in lap swimming and long distance running. ${ }^{8}$

Regulating breath by deep breathing nurtures qi. However, improper deep breathing can affect fangsong and qi. In taking a deep breath, the ribcage is often raised, bracing up the chest, which causes tenseness. Taking a deep breath in a state of anxiety calms the mind and relaxes the body. Deep breathing as well as holding the breath can be disciplined not to be stressful in regulating breath.

There is usually a regimen to regulate breath in qi-energetics practice, to inhale in this movement and to exhale in that. However, Taijiquan does not prescribe a fixed breathing regimen to dictate movements in practice. The many movements of Taijiquan are sometimes practiced in varying tempo, which cannot fit into a strict breathing pattern.
Taijiquan at the beginning adopts "natural breathing" to regulate breath-breathe as the practice demands, like a carburetor, so that breathing can be at ease with no burden. Note that this works well with the slow-motion practice. However, some common rules in breathing guide the practice, such as: exhale when issuing a power action like throwing a punch, and just before, inhale on gathering energy; inhale on raising and exhale on lowering; inhale on opening and exhale on closing, et al.

The rationale of natural breathing in Taijiquan is for the breath to follow the fangsong resolution of nurturing qi. That is, regulate breathing to follow the movements, not the other way around, where breath dictates movements. Let the breath follow the fangsong in the muscle activations at the joints. As qi is nurtured in fangsong together with the constant attentiveness of the breath following the fangsong, the breath becomes adhered to the qi. In other words, after an arduous process of training, the breath qi becomes unified with bioenergy qi in practice, affirming the usage of the same Chinese character for both.

In the fangsong play of the kua, the breath is trained to follow the movements of the diaphragm and the abdominal muscles, and in time, natural breathing develops into abdominal breathing. However, to reemphasize, the practice is mindful not to let the abdomen contract fully like a bellows, which would then hollow out the midsection, causing internal imbalance. At the more advanced level of practice, the abdominal breathing develops into "reverse abdominal breathing," which gives versatility to abdominal contraction to maintain qi connection to the legs. This qi connection is consolidated by the breath following the fangsong of the hip-joints and feet, which leads to "breathing with the heels," as noted by the Taoist sage, Zhuangzi (361 - 296 B.C.). ${ }^{9}$

\section{The tao of fang song}

Getting back to the pragmatics of fangsong, the soft yin-yang logic does not lend to reductionism that offers quantitative measures of values in balance. And the fangsong tool can only be applied to discern imbalance and to reduce the errors of excessiveness (yang) or deficiency (yin). How does Taijiquan practice ensure that the fangsong process leads to a path of convergence to inner balance?

Two crucial factors of fangsong must be developed to lead to convergence. One, fangsong is an organic tool, so must be refined continually to penetrate the ever deeper vestiges of the errors of muscle actions. And two, the attentiveness to the practice must develop as meditation to inspire the increasing concentration needed in the fangsong operation. The meditative component becomes more important as the practice advances and its development is necessary to sharpen and refine the fangsong tool. ${ }^{10}$

The good news in this regard is that Taijiquan is far easier in practice than in theory. You simply apply fangsong to resolve imbalance as it is perceived. The methodology only requires that you stay in the middle ground between not too yin and not too yang. For that, you have to be constantly mindful and attentive, so that you are aware of the errors and their resolution by fangsong with the concomitant qi.

In practice the theory unfolds thus: At the advanced stages, when qi is sufficiently developed, the fangsong tool relies more on qi as a medium to discern and resolve the imbalances. Gradually the qi is used to modulate motion. The stronger qi translates as an indicator of more aligned and balanced muscle activations. The recipe of attentive meditation is the refining of the fangsong tool to get at the subtler errors. In this way, by staying in the "middle ground," as the margin of errors tapers under the ever sharpening fangsong tool, 
the path eventually converges to inner balance. This represents the quintessence of the Tao of "doing without doing" (wei wu wei $\square \square \square$ ).

In the meanwhile the yi-qi-motion paradigm is realized in the maturity of qi development: The yi-command stirs the qi; the qi dynamics drives the motion, forging the unity of qi dynamics (internal) and motion (external). This represents the unity of the internal and external-nei wai jie he $\square \square \square \square$. In this way, the yi-command at the top hierarchy transmits via qi to muscle innervation at the bottom hierarchy in the discipline of Taiji motion.

\section{The kua junction and dantian centrality}

The hard part really is not the science, but the training to deliver the goods of neijin. We have "hundreds of joints" and checking through the imbalances at each one of them is clearly formidable enough as an undertaking, but what makes it nigh insurmountable is that resolving the errors at one joint requires recalibration at the other joints because of the tensile integrity of the body frame.

Ironically, while the problem is one of systems science, we again find a practical solution in the same traditional theory of yin and yang and qi. Guided by the Principle of Three Sections and the Principle of Three Harmonies, ${ }^{11}$ the complex of joints are divided into sections or correspondences of three for the fangsong resolution to work through systematically, and then through further subdivisions in refinement. But more than a scheme of simplification, the principles guide the transmission of motion through the joints and build the harmony of the correspondences of joints.

For instance, the fangsong in the correspondence of the shoulder and kua aligns and balances the internal components of the torso, thus harmonizing its internal motion and momentum. In an example of the three-section classification, the leading direction of the hand (as the extremity section) induces the driving force at the shoulder (as the root section), to transmit smoothly and timely through the elbow (the middle section). The theories are embodied in the Ten Essential Principles of Taijiquan by Chen Changxin (1771-1853), the 14th Generation Patriarch of the Chen Family Taijiquan, and are discussed more fully in the author's book. ${ }^{12}$

In essence, the principles reduce the issues of the myriad joints to the kua junction serving as a base of reference in the fangsong resolution. This reinforces the eminent status of the kua junction as the division between the upper and lower body in generating waistgroin power, discussed earlier. In practice, it means that the fangsong resolution involves a continual play of muscle actions of the pelvic platform and of the SIJ and the hip joints (Figure 2).

More importantly, the constant reference to the kua is nurturing a centrality of motion at the midpoint of the junction. This midpoint coincides functionally with the dantian $(\square \square)$ location, which is also at the same level of the SIJ. The principle of the centrality of the dantian affirms the SIJ as the hub of the transfer of forces between the three levers, the spine of the upper-body and the legs of the lower body.

From the qi perspective, the referencing to the dantian in fangsong creates a qi connectivity between the joints and the dantian, and thus a web of qi connectivity centered at the dantian. The upper-limb movement is always qi-connected through the dantian to the legs anchoring on the ground via a path of qi dynamics.

In other words, the fangsong resolution is cultivating qi that accentuates the centrality of the dantian-filling up the lower abdomen with qi and breath qi and concentrating at the dantian, which is appropriately called dantian qi. Thus, the practice of fangsong of the myriad joints to attain inner balance is reduced to cultivating the fullness of dantian qi to establish the centrality of the dantian. ${ }^{13}$

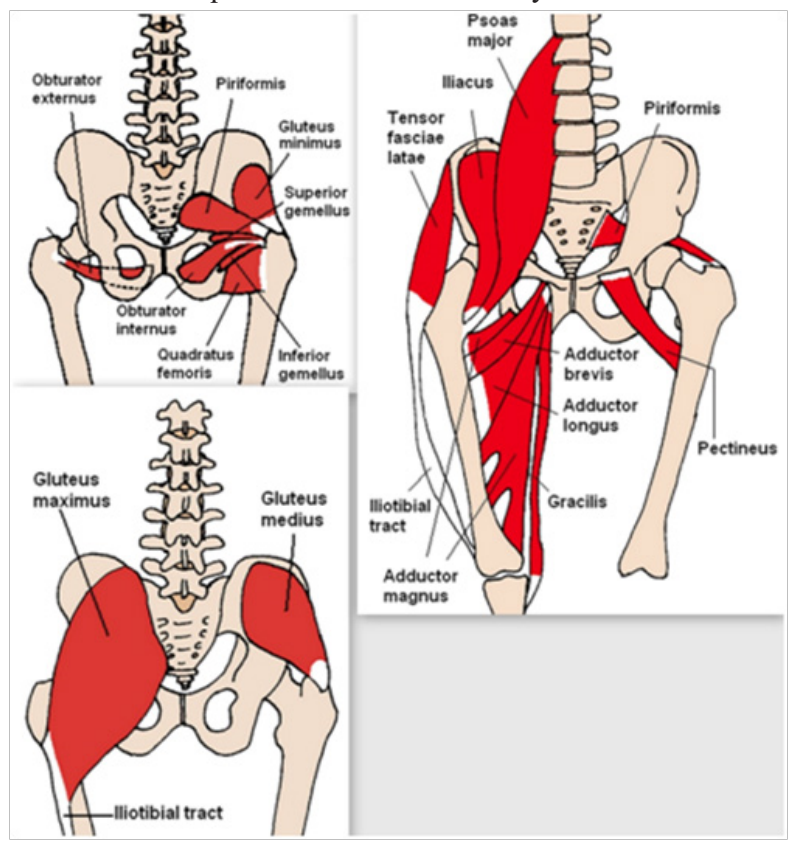

Figure 2 The muscles of the pelvic platform and the three levers.

The fullness of dantian qi signifies that the central status of the dantian is formed (yi dantian wei hexin xing cheng $\square \square \square \square \square \square \square \square$ ) and represents the mastery of the art. The establishment of dantian centrality means that in the yi-qi-motion paradigm, the guiding qi is the dantian qi. This brings to fruition the central control at the dantian and inner balance is achieved. Qi dynamics and motion-internal and external — are unified in accord with the Taiji principles. Neijin is born of this motion.

\section{Explosive release of energy-fajin}

A most illustrative manifestation of neijin is the fajin $\square \square$ action. Fajin is an explosive release of energy in an action. When the body's entire energy is harmonized and directed in the release, the power of the fajin is mesmerizing and never fails to impress. The crisp power of Chen Xiaowang's signature fajin exemplifies the unison of body motion and momentum, as captured in the video link. ${ }^{14}$

Let us revisit the biomechanics of dang-yao jin (waist-groin power) to review neijin in the fajin of a punch. The two crucial factors in the execution are: 1) the mutual support between the upper and lower body motion and 2) the body's rotational motion. At the kua junction, both factors are integrated to produce the torque action and reaction in mutual support and balance.

The mechanics of rotating the torso at the spinal axis seems easy enough, but the torso is not a simple solid piece. Structurally, the torso consists of the top-heavy ribcage hanging on the upper part of the vertebral column, which lower end, the sacrum, sits precariously at the pelvic base. The structure may fit perfectly in the musculoskeletal framework, but it is inherently unstable. Unsound as the skeletal structure may be, dressed up in muscles, it is an engineering marvel that gives us our remarkable bipedal functionality and balance. Nevertheless, it is very tricky to exploit the engineering design to deliver the power of neijin.

We want the torso to move as one unit in the action, but often the left and right sides of the body do not act in sync. For instance, in 
the anxiety to strike out fast, in say a right-hand punch, the right side tends to dominate, which causes a drag on the left part of the body. Golf has a simple exercise to condition the left and right sides to move in sync - turning the body with a broomstick held against the back.

We also want the torso to turn at the kua junction, but we are predisposed by our walking gait to turn at the chest level (at the T12/ L1 vertebrae). As noted earlier, this would deprive the torso of the momentum of the mass of the midsection. The rotational problem is compounded by the torsion introduced in the spine by lateral flexion due to the spinal curvatures. ${ }^{15}$

These issues are resolved by Taijiquan's cultivation of inner balance. The fangsong applied to the shoulder-kua correspondence works to balance and align the many muscles of the torso (the lats, gluts, pecs, deltoids, traps, et al.) with the core axial muscles that maintain the integrity of the spine. The discipline of inner balance unifies the internal movements of the torso, thus its internal momentum.

We can now appreciate the gruesome demand of the practice of stationary horse stance in Chinese Martial Arts, a basic training to settle the torso into the kua. The practice of throwing the same punch thousands of times and of standing stationary in a horse stance in the years of kungfu training are to connect the shoulder and kua to turn as a unit in motion, thus packing the punch or action in unified momentum without losing balance and control.

The practical import of the continual fangsong play of the kua to establish dantian centrality is to facilitate the ease of motion and force transmission at the SIJ hub and hip-joints via the pelvic platform. Incorporated in the control of the pelvic platform is the discipline of the spinal torsion due to the spinal engine, thus harmonizing the axial angular momentum. ${ }^{16}$ This harmony is necessary for the spine to unify with the kua's rotation to produce maximal dang-yao jin (waist-groin power).

The principle of inner balance is indeed comprehensive: It endows integrity of structure and balance and alignment of muscle actions at the joints. Driven by the dynamics of dantian qi, the internal movements of the segments are forged in unity. With the principle of harmony, the internal motion flows smoothly through the body unforced, with no obstruction; the left and right in sync, the upper and lower body are balanced in mutual support. This harmony is the fruition of the internal dimension of the Principle of Three Harmonies: the harmony of the heart (xin $\square$ ) and mind (yi $\square$ ), physical force ( $l i \square)$ and qi ( $q i \square$ ), and tendons-muscles (jin $\square$ ) and bones ( $g u \square$ ).

When motion regulated by inner balance is accelerated, what ensues is a refined and cultured expression of force that is explosivefrightening and graceful at the same time. Guided at the dantian hub, the issuance of fajin flows forth balanced and unforced, so appears effortless with no visible exertion. Grounded on inner balance every muscle fiber and sinew are aligned in the fajin action. Not burdened by flaws of imbalance, the full potential of the body's energy is directed at the action, so that the fajin power delivered is consummate.

Actually, fajin is a simple action like stepping on the gas pedal However, if the body is not Taiji regulated, it would be like a car not in tune, wheels unaligned, and tires uneven, sputtering off the road. Imbued with inner balance, the fajin motion transmits with no resistance through the body and emanates at the extremity like the crack of a whip, with the silk garment snapping crisply on the player's body.

The fajin action manifests the gang (hardness) yang character of neijin, where the limbs and body are summoned to move in unison, including the internal motion of the organs, tissues and fascia, thus bringing all the momenta to bear in unity. There is an opposite yin, the rou (softness) of neijin, which characterizes the ease of change of the internal movements of the body, thus the liveliness of maneuverability.

\section{The rou (soft) and gang (hard) of neijin}

The reason why the traditional Taiji theory works in the science of body motion is that the body responds to the organic logic of yin and yang. The body uses the yin-yang duality to access the force of neijin.

Force is a vector with two components, magnitude and direction. Our neurobiology sees and responds to force not in quantitative but relative terms. The body does not produce force vector of a desired magnitude and direction, but responds in interactions which results in a force, which may or may not be sufficient. For instance, when pushed in a tussle, we respond instinctively by pushing back to prevent from being shoved off and to fight back. This relies only on one component of the force, namely, its magnitude. And we know well that in a contest of strength, the stronger force would prevail in a linear dimension. The "natural" response of pushing back clearly is a poor strategy for a person of weaker disposition. We can mitigate the force's impact if we can maneuver the body to absorb and receive the incoming force at an oblique angle. But, the problem is that, once the muscle activations are committed in the response, the body tends to be locked in the posture, rendering it unable to change.

Neijin responds with soft (rou $\square$ ) and hard (gang $\square$ ) to result in the right force vector to counter the attacking force. The rou response allows the body to adjust internally to absorb and thus withstand the force at a reduced magnitude of impact. The maneuver uses the lively (linghuo $\square \square$ ) path of qi-connectivity centered at the dantian to do so, which is accorded by the Principle of Dantian Centrality. This is the skill of "using softness to overcome hardness" (yi rou ke gang $\square$ $\square \square \square)$.

Neijin's strategy is to use the rou response to let the opponent's aggressive force be spent, and then follow with timely gang response to repel the attack with the formidable force that flows from unified internal momentum. In the rou response, there must be sufficient $g$ ang to support it, otherwise one would have been shoved off before any technique could come into play, and in the gang response, there must be rou to maneuver to accommodate unexpected changes to the load on the body. Most crucial of all, the principle of inner balance accords maneuverability in the instantaneous change between rou and gang, and maintains balance at all times.

The oft touted classic kungfu skills of Taijiquan. ${ }^{17}$

"Four ounces repel a thousand pounds" Si liang bo qian jin $\square \square$ $\square \square \square$ “Lead opponent's force to emptiness" Yin jin luo kong $\square \square \square \square$

““"Borrow force to strike back” Jie li lai da ren, $\square \square \square \square \square$

and the qinna $\square \square$ joint-locking skills, all rely on the liveliness of the change of rou and gang of neijin in the application..

In short, the magic of the consummate force of neijin lies in the response of the right measures of rou and gang that imparts the rou liveliness in the interchange, and the gang explosiveness of power in the unified momentum.

The good news for a golfer is that the will no longer be elusive. The buildup of neijin in Taijiquan's practice can always be tapped upon to produce the waist power needed in the swing.

\section{The triple gems}

The achievement of Taiji balance does not bestow just one gem, but the triple gems: 


\section{i.Neijin \\ ii. Equanimity \\ iii. Homeostasis}

When you attain inner balance in Taijiquan training, you are rewarded with neijin and all the kungfu skills that flow from it. But the prize is far greater. You gain bliss and tranquility of mind, and something else, you also get the package that provides health and wellbeing, namely, the homeostasis of the biological and physiological environment of the body.

Although we have mentioned meditation only briefly, its development is complementary in Taijiquan practice. Fangsong resolution requires constant attentiveness to the process, which is the mind aspect of the practice. It has the effect of restraining the restlessness of the "monkey mind," and thus quieting the mind. Calmness is conducive for the mind to stay focused, which reinforces attentiveness that further cultivates awareness and concentration. The discipline builds a sea of tranquility in the mind and one enjoys bliss and equanimity. More of meditation and Taijiquan can be found in the author's book. ${ }^{18}$

In TCM, qi and blood are inextricably intertwined in concept and function. Sometimes blood is described as "a denser form of qi." TCM sums it up thus:

Qi is the commander of blood.

Blood is the mother of qi.

In this qi-blood inseparability, TCM's central tenet of qi as the life-force energy that gives life and vitality in biology is easier to appreciate as blood circulating the body delivering oxygen, nutrients, ions and hormones.

The qi-blood inseparability is borne out in Shin Lin's research, which shows that not only are blood flow and electricity increased but that the increases are coordinated in Taijiquan and qigong, ${ }^{19}$ as well as in other Chinese therapies such as acupuncture, massage, and topical herbal remedies. ${ }^{20}$

Thus, by TCM's qi-blood theory, the fullness of qi developed in inner balance inures to the blood in all its optimality to benefit the body's biology. In other words, inner balance delivers the full package of homeostasis to every system of the body's physiological environment. It should also be mentioned, peripheral to blood flow, Taijiquan's deliberative slow motion in fangsong supports the transport of lymph up the body in the circulation of the lymphatic system. The fangsong and muscle activations provide a coherence of pressure at the lymph nodes, particularly at the feet and at the inguinal fold where there is a concentration of.

\section{Biography}

C.P. Ong is the Executive Vice-President of the USA WushuKungfu Federation. He is a 20th generation Chen Family Taijiquan disciple of both Chen Xiaowang and Chen Zhenglei. He has also studied extensively with Zhu Tiancai as well. He first began his Taiji studies in 1972 learning the Guang Ping Yang Style from Master Y.C. Chiang in Berkeley, CA. He is also a student of vipassana (insight) meditation and has attended several intensive meditation retreats in Buddhist monasteries in Yangon, Myanmar.

He received his Ph.D. in Mathematics from U.C. Berkeley in 1973.

\section{Acknowledgments}

None.

\section{Conflicts of interest}

Author declares there are no conflicts of interest.

\section{Funding}

None.

\section{References}

1. The five classics are: Taijiquan Treatise (attributed to Zhang Sanfeng), Taijiquan Classiscs (by Wang Zongyue, Thirteen Postures (Anonymous), Elucidation of the Thirteen Postures (by Wu Yuxiang) and Song of Pushhands (Anonymous).

2. Vleeming A, Schuenke MD, Masi AT, et al. The sacroiliac joint: an overview of its anatomy, function and potential clinical implications $J$ Anat. 2012;221(6):537-567.

3. Serge Gracovetsky. Spinal Engine Theory. Springer-Verlag, New York, USA. 1989.

4. CP Ong (Draft) The Spinal Engine and Taijiquan.

5. CP Ong. Generating Inner Strength Through Taijiquan. Int J Complement Alt Med. 2016;3(3):00076.

6. Shin Lin .Research Leading to Systems/Cellular/Molecular Model for the Benefits of Qigong and Tai Chi on Health and Healing. Theses: Scientific and Skill Papers on Qigong, The World Qigong Forum 2007 and 10th World Congress on Qigong and Chinese Medicine, Tokyo, Japan. 2007.p.3-8.

7. Shin Lin Electromyographic study on Tai Chi's activation of muscles essential for fall prevention and weightlifting. $J$ Altern Complement Med. 2013;19:A49.

8. www.HumanKinetics.com

9. CP Ong .More of abdominal and reverse abdominal breathing. Cultivating Inner Strength. Bagua Press, China. 2013.p.257.

10. Ibid 9. Role of Meditation in Taijiquan. pp.264.

11. This consists of Three External and Three Internal, making it the Six Harmonies, as it is also known.

12. Ibid 9. The principles are discussed in Chapter 7.

13. CP Ong .16) The Central Status of the Dantian. Tai Chi Magazine. $2016 ; 4(1)$

14. https://www.youtube.com/watch?v=5LosS2vjmek

15. Ibid 3. Gracovetsky's Spinal Engine Theory.

16. Ibid 4.

17. Ibid 5. Discussed in the Section: The Physics of the Prowess of Neijin and also in author's book.

18. Ibid 9. Meditation and Perceptiveness. p. 266

19. Lin, G Orenstein G, Froloff A, Nguyen N, et al. P01.29. Pre-polarization conductance at Jing-Well acupoints on the hand is correlated with blood flow measured by laser doppler flowmetry. BMC Complement Altern Med. 2012;12(Suppl 1):P29.

20. Ross LT, Sutherland N, Orenstein G. P01.05. Increase in blood flow associated with traditional chinese medicine therapies. $B M C$ Complement Altern Med. 2012;12(Suppl 1):P5. 